\title{
Determinanten der Niederlassung als Allgemeinmediziner - eine qualitative Studie
}

\section{Determinants of Setting up a General Practice - a Qualitative Study}

\section{(ㄷ) (1) (우)}

\author{
Autoren \\ Ruben Zwierlein ${ }^{1} \mathbb{D}$, Frank Portenhauser ${ }^{2}$, Kristina Flägel ${ }^{1}$, Jost Steinhäuser ${ }^{1}$
}

Institute

1 Institut für Allgemeinmedizin, Universitätsklinikum Schleswig Holstein - Campus Lübeck, Lübeck

2 Kassenärztliche Vereinigung Baden-Württemberg, Koordinierungsstelle Allgemeinmedizin Baden-Württemberg, Stuttgart

\section{Schlüsselwörter}

Angestellter Arzt, Niederlassung, Hausarzt, Allgemeinmedizin, Praxismanagement

\section{Key words}

Employed physician, Setting up a practice, General practitioner, Family medicine, Practice management

\section{Bibliografie}

DOI https://doi.org/10.1055/a-1076-7848

Online-Publikation: 7.4.2020

Gesundheitswesen 2020; 82: 527-533

(c) Georg Thieme Verlag KG Stuttgart · New York ISSN 0941-3790

\author{
Korrespondenzadresse \\ Ruben Zwierlein \\ Institut für Allgemeinmedizin \\ Universitätsklinikum Schleswig Holstein - Campus Lübeck \\ Ratzeburger Allee 160 \\ 23538 Lübeck \\ r.zwierlein@uni-luebeck.de
}

\section{ZUSAMMENFASSUNG}

Ziel der Studie In den letzten Jahren kann beobachtet werden, dass die Motivation der nachrückenden Fachärzte für Allgemeinmedizin sich in einer eigenen Praxis niederzulassen rückläufig ist. Mit der Kenntnis der hier fördernden und hemmenden Faktoren, können Strategien zur Förderung der Niederlassung abgeleitet werden. Ziel dieser Studie war es daher diese Determinanten zu erheben.

Methodik Es wurden niedergelassene Fachärzte für Allgemeinmedizin aus Baden-Württemberg (BW) im Zeitraum von April bis Oktober 2016 eingeladen, an einer qualitativen Inter- viewstudie teilzunehmen. Die Stratifikation der Ärzte aus einer Gruppe von insgesamt 553, die zwischen 2008 und 2011 am Förderprogramm Allgemeinmedizin in BW teilnahmen und sich in den Jahren 2008 bis 2014 niederließen, erfolgte anhand der Verdichtungsräume im ländlichen Raum, der Randzonen um Verdichtungsräume und der Verdichtungsräume selbst. Die Transkripte der Interviews wurden von zwei Autoren inhaltsanalytisch nach Mayring ausgewertet, zusammengefasst und im zweiten Schritt in eine Konsensversion überführt.

Ergebnisse Es konnten 23 Allgemeinmediziner rekrutiert werden, die zu $57 \%$ weiblich und im Durchschnitt 42 Jahre alt waren sowie mehrheitlich in Kooperation arbeiteten. Hauptmotivation sich niederzulassen lag in dem Wunsch die Situation gegenüber dem Klinikalltag zu verbessern. Hierzu zählten insbesondere eine geringere Dienstbelastung und familienfreundlichere Arbeitszeiten. Relevante, unterstützende Faktoren während des Niederlassungsprozesses waren der familiäre Rückhalt, die (Verbund-)Weiterbildung und professionelle Beratung. Hemmende Faktoren waren Ängste vor Regressen und Verschuldung, das als kompliziert erlebte Abrechnungssystem und der als bürokratisch erlebte Zulassungsprozess.

Schlussfolgerungen Die Studie zeigt zum einen, dass die vielen Vorteile als niedergelassener Allgemeinmediziner, wie z. B. die gute Familienkompatibilität, besser kommuniziert und die finanziellen Bedenken entschärft werden müssen. Zum anderen zeigt sich der Bedarf nach Lehrangeboten in der Ausbildung zum Praxismanagement. Ein standardisierter und optimierter Niederlassungsprozess mit verbesserter Übersicht und einem kollegialen Ansprechpartner kann die Entscheidung für die Niederlassung und die Durchführung erleichtern.

\section{ABSTRACT}

Background In recent years, the motivation of the new generation of general practitioners to set up their own practice is decreasing. Knowledge of factors that promote and inhibit the process of setting up a practice can enable developing strategies to support this process. The aim of this study was to explore these determinants.

Methods Between April and October 2016, self-employed general practitioners from Baden-Württemberg were invited 
to participate in a qualitative interview study. Stratification of 553 physicians, who participated in a general practice support program between 2008 and 2011 and set up a practice in 2008 to 2014 , was based on the agglomeration in rural areas, the peripheral zones around the agglomeration and the urban agglomerations themselves. The transcripts of the interviews were analyzed by two authors with the content analysis according to Mayring and summarized. In a second step, a consensus version was elaborated.

Results A total of 23 general practitioners were recruited, $57 \%$ female and 42 years on average, and mostly working in group practices. The main motivation to set up a practice was the desire to improve the situation in relation to the daily clinical routine. These included a lower duty load and more familyfriendly working hours. Relevant supportive factors during setting up a practice were family support, participation in a postgraduate training rotation network program and professional counseling. Inhibiting factors were fears of recourse and indebtedness, a complicated accounting system and a bureaucratic process of admission.

Conclusions On the one hand, the study shows that the many advantages of working as a general practitioner, such as its being family friendly, need to be better communicated to the doctors and their financial concerns alleviated. On the other hand, the need for courses on management of one's practice has also become clear. A standardized and optimized process of setting up a practice with an improved overview and a collegial contact person can facilitate the decision for and implementing the setting up a medical practice as a general practitioner.

\section{Einleitung}

„Wir haben weniger einen Mangel an Medizinern, sondern vielmehr eine Fehlverteilung, und zwar eine doppelte. Zum einen nimmt die Zahl der Hausärzte ab und die der Fachspezialisten stark zu. Die zweite Fehlverteilung ist die regionale. Die meisten Ärzte praktizieren dort, wo sie am wenigsten gebraucht werden, in den gut situierten Bezirken unserer Großstädte. In Stadtteilen mit wenig Privatpatienten und niedrigem sozioökonomischem Status gibt es schon weniger Ärzte. Und besonders prekär ist es aufdem Land, wo wir in der Zukunft eine echte Gefährdung der Grundversorgung erwarten“. (Vorsitzender des Sachverständigenrats zur Begutachtung der Entwicklung im Gesundheitswesen Professor Dr. med. Ferdinand Gerlach aus dem Jahr 2011) [1].

Noch nie gab es so viele Ärzte in Deutschland wie heutzutage. Gleichzeitig ist der Anteil an angestellten Ärzten, die keinen vollen Versorgungsauftrag übernehmen so hoch wie noch nie [2]. Der Blick allein auf Köpfe birgt die Gefahr die abfallende Tendenz gemessen am Teilnahmeumfang der Ärzte zu übersehen. Dieser wird in Vollzeitäquivalenten (VZÄ) angegeben und ist das Verhältnis zwischen gearbeiteten Stunden und üblicher Arbeitszeit einer Vollzeittätigkeit [3].

Lag 2009 das VZÄ eines durchschnittlich Arztes in der vertragsärztlichen Versorgung bei 0,98, fiel es im Jahr 2017 auf 0,88 [4]. Grund hierfür ist hauptsächlich der gestiegene Anteil an angestellten Ärzten in der vertragsärztlichen Versorgung (23,1 statt 7,3\%) [4-6]. Das VZÄ lag 2017 bei einem niedergelassenen Vertragsarzt bei 0,93 und bei einem angestellten Arzt bei 0,68. Zudem wird auch die vertragsärztliche Versorgung zunehmend weiblicher. Eine Ärztin hatte 2017 im Schnitt einen VZÄ von 0,85, ein Arzt von 0,91 [4].

Vergleichbares gilt für den hausärztlichen Bereich. Die Anzahl an Facharztanerkennung für Allgemeinmedizin stieg in den letzten acht Jahren kontinuierlich an [7]. Von 2013 bis 2017 hat sich der Anteil an angestellten Ärzten mit 19,5\% in der hausärztlichen Versorgung fast verdoppelt. Das VZÄ betrug 2017 bei niedergelassenen Hausärzten 0,99 und bei angestellten Hausärzten 0,77 [6]. Diese Reduktion des VZÄ eines durchschnittlichen Hausarztes um zwei Prozentpunkte entspricht auf die gesamte Hausärzteschaft übertragen über 1000 Hausärzte weniger [5, 6]. Eine ähnliche Ten- denz auf Ebene des zukünftigen Bedarfs an hausärztlicher Versorgung auf Gemeindeebene zeigte eine Studie aus dem Jahr 2015 [8].

Aufgrund des bestehenden Geburtendefizits und der unzureichenden Nettozuwanderung prognostizierte das Statistische Bundesamt im Jahr 2015 eine Verdoppelung des Anteils der Menschen im Alter von 65 Jahren oder mehr von 16\% im Jahr 2013 auf rund $33 \%$ im Jahr 2060 [9]. Diese Entwicklung lässt sich bei den Allgemeinmedizinern mit einem Anteil an über 65-jährigen 2011 von 8 auf $16 \% 2017$ ebenfalls nachvollziehen [10,11]. Aufgrund des Alters wird in dieser Gruppe Nachfolge gesucht. Zusätzlich zur demographiebedingten, steigenden Nachfrage an Nachfolgern haben Ärzte im ländlichen Raum Mühe, einen Nachfolger zu finden. Fast 50 \% der nachrückenden Ärztegeneration gaben in einer Befragung aus den Jahren 2014 und 2018 eine „Aversion“ gegen das Arbeiten auf dem Land an [12]. Die prognostizierte Wiederbesetzungsquote im Bereich Allgemeinmedizin liegt bei $47 \%$, rund jede zweite Allgemeinarztstelle würde demnach wiederbesetzt werden. Diese Modellrechnung basiert allerdings auf Präferenzen von Studierenden im Praktischen Jahr und setzte diese in Verhältnis zur relativen Häufigkeiten der jeweiligen Facharztzahlen [12].

Fördernde und hemmende Faktoren (Determinanten) zu erheben, um eine maßgeschneiderte Intervention durchzuführen ist eine Methode, die aus der Implementierungswissenschaft entnommen wurde. Hierbei erfolgt zunächst eine Problemanalyse mit angeschlossener Identifikation und Priorisierung von Strategien [13].

Ziel dieser Studie war es Determinanten einer Niederlassung als Allgemeinmediziner zu explorieren, um daraus Strategien gegen den Hausärztemangel abzuleiten.

\section{Methodik}

Grundlage der Untersuchung sind Telefoninterviews mit Fachärzten für Allgemeinmedizin anhand eines semi-standardisierten Leitfadens, welche im Institut für Allgemeinmedizin in Lübeck im Zeitraum von April bis Oktober 2016 durchgeführt und transkribiert wurden. 


\section{Rekrutierung}

Von den 553 Fachärzten für Allgemeinmedizin, die zwischen 2008 und 2011 am Förderprogramm Allgemeinmedizin teilnahmen und sich in den Jahren 2008 bis 2014 niederließen, wurden 150 anhand der Zuteilung nach Verdichtungsräumen im ländlichen Raum, Randzonen um Verdichtungsräume und Verdichtungsräume stratifiziert und in zwei Phasen angeschrieben. Zusätzliche Kriterien waren die Art und Dauer der vertragsärztlichen Tätigkeit: Zulassung und mindestens zwei-jährige Tätigkeit als niedergelassener Arzt. Ein Reminder wurde nicht versendet.

Zur Klassifizierung der Raumregionen wurde der Landesentwicklungsplan des Ministeriums für Wirtschaft, Arbeit und Wohnungsbau Baden-Württemberg verwendet [14]. Das Geschlechtsverhältnis bei der Zulassung betrug $60 \%$ weibliche und $40 \%$ männliche Ärzte, sodass auch in diesem Verhältnis rekrutiert wurde. Hinsichtlich der Zulassung in städtischen und ländlichen Regionen wurde ein Verhältnis von 50:50 ausgewählt.

Das Förderprogramm Allgemeinmedizin wurde 1998 mit der Schaffung des Gesetzliche Krankenversicherung-Solidarisierungsstärkungsgesetzes im Artikel 8 ins Leben gerufen. Es war zunächst eine reine finanzielle Förderung, seit 2010 beinhaltet sie auch strukturelle Elemente [15].

Im Rahmen der digital aufgezeichneten Telefoninterviews (KF) wurden unter zur Hilfenahme eines semistrukturierten Leitfadens sieben Fragen zu Gründen für eine Niederlassung, dem Prozess der Niederlassung, den Auswahlkriterien für eine Praxis und dem Prozess nach der Niederlassung gestellt.

Während oder kurz nach der Aufzeichnung wurden Gesprächsprotokolle mit den wichtigsten Punkten angefertigt (KF).

Die Leitfragen wurden zum einen auf dem Boden einer pragmatischen Literatursuche, die mit den Suchbegriffen „career choice“ AND „family practice“ AND „Germany“ durchgeführt wurden [1619] erstellt. Des Weiteren wurden die Erfahrungen einiger der Autoren (Leiter einer Koordinierungsstelleeiner Kassenärztlichen Vereinigung (KV), eine Ärztin in Weiterbildung zum Facharzt für Allgemeinmedizin und ein habilitierter Facharzt für Allgemeinmedizin mit Forschungsschwerpunkt zu Strategien gegen den Hausärztemangel) mit einbezogen.

Die pseudoanonymisierten und transkribierten Interviews wurden im ersten Schritt von zwei Autoren (RZ \& FP) unabhängig voneinander nach der strukturierten qualitativen Inhaltsanalyse von Mayring ausgewertet und zusammengefasst. Hierzu wurden zunächst Kategorien gemäß der Leitfragen erstellt und anschließend induktive Kategorien aus den Transkripten abgeleitet [20]. Im zweiten Iterationsschritt wurde eine Synopse der beiden unabhängigen Zusammenfassungen von RZ erstellt und in Zusammenarbeit mit FP und JS in eine Konsensversion überführt. Während des ganzen Prozesses fungierte JS als Supervisor. Er hat bereits mehrere qualitative Studien durchgeführt.

Die Transkripte wurden den teilnehmenden Ärzten nicht zum Korrekturlesen vorgelegt und es wurde kein Ergebnisfeedback von ihnen eingeholt.

Die Konsensversion bildete die Grundlage der hier präsentierten Ergebnisse.

Im Folgenden stellen wir die Ergebnisse der Fragen, wie der Entscheidungsprozess zur Niederlassung verlief, warum man sich niederlassen wollte und wie sich der Niederlassungsprozess gestaltete, dar.

\section{Ethik}

Dieser Studie liegt die Einwilligung jedes geförderten Arztes in Weiterbildung nach $\S 8$ der Bundesvereinbarung zur Evaluation des Förderprogramms zugrunde. Die Teilnahme an der Studie war freiwillig. Durch die Beteiligung des Instituts für Allgemeinmedizin war eine Zuordnung der Teilnehmer für die Kassenärztlichen Vereinigung BW nicht möglich. Die Einwilligung in die Studie konnte jederzeit zurückgezogen werden.

Ein Ethikvotum wurde daher nicht gesondert eingeholt.

\section{Ergebnisse}

\section{Demografie}

- Tab. 1 gibt eine Übersicht der Soziodemografie der Stichprobe. Von den 23 befragten Ärzte waren $57 \%(n=13)$ weiblich und $52 \%$ ( $n=12$ ) arbeiteten im ländlichen Raum (15\% Rücklaufquote). Die Mehrheit arbeitete mit mindestens einem Arzt in der Praxis zusammen $(70 \%, n=16)$. Der durchschnittliche Befragte war 42 Jahre alt und hat seit 14 Jahren das Studium abgeschlossen. Ein Interview dauert im Durchschnitt 18 Minuten (min. 7, max. 84).

\section{Gründe sich niederzulassen}

Die Gründe für eine Niederlassung waren vielschichtig. Sie ließen sich in private und berufliche Aspekte unterteilen. Im privaten Bereich nahm die Familie einen wesentlichen Raum ein. Zentrale Punkte im beruflichen Bereich stellten die Unzufriedenheit mit dem Klinikalltag und dem Angestelltenverhältnis sowie positive Erfahrungen im Rahmen der Weiterbildung dar. Ein weiterer Faktor bestand in der Prägung durch niedergelassene Eltern bzw. Großeltern.

Familienkompatibilität

Niedergelassen zu sein bot mehr Zeit für Familie. Insbesondere gaben Frauen ein hohes Maß an Flexibilität an und einen vereinfachten Wiedereinstieg nach der Familiengründung. Ein wesentlicher Punkt war die gute Vereinbarung von Beruf und Familie.

„[...] und dann musste ich einfach nach was schauen, was A zu meinem medizinischen Profil passt, also zu dem was ich gerne mache, und B familienkompatibel [...]“. (NL1, Z. 14 ff.)

Tab. 1 Soziodemografische Daten der Teilnehmer $(N=23)$.

\begin{tabular}{|l|l|l|}
\hline \multicolumn{2}{|l|}{ Soziodemografische Variablen } & N (\%) \\
\hline \multirow{2}{*}{ Geschlecht } & Männlich & $10(43,5 \%)$ \\
\cline { 2 - 3 } & Weiblich & $13(56,5 \%)$ \\
\hline \multirow{2}{*}{ Praxisform } & Einzelpraxis & $6(26,1 \%)$ \\
\cline { 2 - 3 } & Mehr als ein Arzt & $16(69,6 \%)$ \\
\hline \multirow{2}{*}{ Praxislage } & Stadt & $10(43,5 \%)$ \\
\cline { 2 - 3 } & Land & $12(52,2 \%)$ \\
\hline Alter (in Jahren) & & MW [min.; max.] \\
\hline Zeit seit Studienabschluss (in Jahren) & $14[6 ; 26]$ \\
\hline Niedergelassen seit (in Jahren) & $4[0,25 ; 7]$ \\
\hline Interviewdauer (in Minuten) & $18[7 ; 84]$ \\
\hline$*$ n variiert aufgrund fehlender Daten; MW Mittelwert \\
\hline
\end{tabular}


Gute Erfahrungen in Aus- \& Weiterbildung

Im Rahmen des Studiums oder während der Weiterbildung dominierten positive Erfahrungen in Praxen. Die Niederlassung als Allgemeinmediziner war auch nach einer Phase des Ausprobierens oder beim Wechsel des Fachgebiets beliebt. Zusätzlich hatten Vorbilder wie der eigene Hausarzt, der Weiterbilder oder Kollegen einen prägenden Einfluss.

„Ein super Team, tolle Atmosphäre. Und das Arbeiten an sich hat mir auch Spaß gemacht und dann hab' ich mich entschieden das zu machen “. (NL8, Z. 19 ff.)

„Also das Entscheidende für mich war die Weiterbildungszeit, muss ich sagen “. (NL4, Z. 92 f.)

Unzufriedenheit mit den Arbeitsbedingungen des Klinikalltags

In der Klinik führten die Arbeitszeiten, Nacht- und Wochenenddienste zu Unzufriedenheit mit der Tätigkeit. Der Alltag im Klinikum stellte eine große Herausforderung für die Vereinbarkeit von Familie und Beruf dar. Abträglich war zudem die eingeschränkte Entscheidungsfreiheit. Ein weiterer Aspekt war die unzureichend umgesetzte Weiterbildung. In manchen Fachrichtungen, wie zum Beispiel in der Chirurgie, war der persönliche Alltag stark gefährdet, wenn beide Ehepartner gleichzeitig im Krankenhaus arbeiteten. Unzufriedenheit herrschte ferner gegenüber den hierarchischen und starren Strukturen eines Klinikums.

„Weil ich mir im Prinzip nicht vorstellen konnte, als ich im Krankenhaus war, dauerhaft im Krankenhaus zu arbeiten. Von den Arbeitszeiten nicht, keine Vereinbarkeit von Familie und Beruf, nicht die Möglichkeit selber zu entscheiden... Also die Faktoren kamen zusammen, dass ich gedacht habe, nein, also Krankenhausärztin möchte ich nicht sein [...].“(NL6, Z. 18 ff.)

Der Wunsch nach Selbständigkeit

Neben der im vorherigen Punkt erwähnten Unzufriedenheit mit der beschränkten Entscheidungsfreiheit fanden sich weitere Elemente im Anstellungsverhältnis. Hierbei wurde einerseits die diskontinuierliche Patientenbindung aufgezählt. Andererseits wurde die eigene Arbeit mehr als Dienstleistung empfunden. Zudem lag eine Quelle für Unzufriedenheit darin als angestellter Facharzt wegen unzureichender therapeutischer Freiheit das Gefühl zu haben, weiterhin als Arzt in Weiterbildung zu fungieren. Die fehlende Selbstständigkeit und freie Gestaltung im Arbeitsalltag, die Personalführung, die Arbeits- und Urlaubszeiten sowie die langfristige Planung stellten weitere Gesichtspunkte dar.

„Die Selbständigkeit, eigener Chef sein - ja, selber das Ruder so ein bisschen in der Hand zu haben, keine weiteren Vorgesetzten und letztlich war es auch der Beruf als Hausarzt.“ (NL5, Z. 19 ff.)

\section{Bessere Verdienstmöglichkeiten}

Als niedergelassener Arzt verdiente man mehr als in einer Anstellung.

„Zumal man auch sagen muss, dass die Bezahlung als angestellte Ärztin doch relativ gering ausfällt". (NL15, Z. 31 f.)

\section{Einflussfaktoren auf den Niederlassungsprozess}

Positive Faktoren

Fördernde Faktoren im Prozess der Niederlassung waren

- die im Vergleich zur Klinik selteneren Bereitschaftsdienste,
- die Unterstützung durch Berater (Bank-, Fachanwalt-,

Steuerberater und Berater der Kassenärztlichen Vereinigung),

- der familiäre Rückhalt, insbesondere der Ehepartner und bereits niedergelassener Familienmitglieder,

- die Akzeptanz und die Dankbarkeit der Patienten,

- der kollegiale Austausch,

- Schulungstag im Rahmen der (Verbund-)Weiterbildung.

„Die Bedingungen haben gepasst, über das Reingucken können in die Praxis, die Weiterbildungszeit hatte ich erlebt als intensiv und auch als bestätigende, die Bank hat mich unterstützt, die Familie hat mich unterstützt, die KV hat mich unterstützt [...]“. (NL4, Z. 455 ff.)

Negative Faktoren

Hemmende Faktoren im Prozess der Niederlassung waren

- die komplexe Abrechnung,

- unzureichende Vorbereitung auf die Abrechnung,

- finanzielle Themen wie Regressangst, die hohen Beraterkosten, die unklare Kosteneinschätzung, die hohen Kosten für Immobilien und Lebenserhaltung in der Stadt, der Aufkauf eines Kassensitzes sowie die Ungewissheit der Einnahmen,

- die Abstimmung mit dem Praxispartner, insbesondere der erhöhte Zeitaufwand und Konflikte bei der Kosten- und Gewinnverteilung,

- der lange Zulassungsprozess,

- die Probleme beim Abgabeprozess der Praxis, wie z. B. ein nicht informierter oder demotivierter Vorgänger.

„Die ganzen Zulassungen [...]. Das war schon etwas bürokratisch.“ (NL12, Z. 33 ff.)

„Das eine ist die finanzielle Seite, die da so auf einen zukommt, mit Startkapital, was ja doch je nachdem ein ordentlicher Batzen ist." (NL19, Z. 59 ff.)

„Immer diese Angst, ob man alles richtig macht, ob vielleicht doch irgendwann ein Regress einem droht [...]. “ (NL6, Z. 57 f.)

Neutrale Faktoren

Zusätzliche Bedenken im Prozess der Niederlassungen waren

- die örtliche Bindung,

- die erhöhte Verantwortung

- und der geringere kollegiale Austausch im Gegensatz zur Arbeit im Krankenhaus.

\section{Auswahl der Praxis}

Die Auswahl der Praxis erfolgte über 3 Wege:

- Vergleichen vieler Praxen

- Praxisübernahme innerhalb der Familie

- Im Rahmen der Weiterbildung

In der Weiterbildungszeit gab es die Möglichkeiten des Einstiegs in die Weiterbildungspraxis, den Kontakt über die Verbundweiterbildung, Tipps und Tricks vom Weiterbilder, einen schrittweisen Übergang z. B. über eine temporäre Anstellung sowie finanzielle Einblicke. Die Kriterien für die Praxisauswahl sind in > Tab. 2 ersichtlich.

„Mein Chef [...] hatte mir auch ein bisschen die Angst genommen, was das Finanzielle angeht [...], hatte ich viele Einblicke gekriegt, was so die Wirtschaftlichkeit der Praxis angeht. Ja, und dadurch war mir ei- 
Tab. 2 Praxisauswahlkriterien sowie Vorteile einer Einzelpraxis und einer Kooperation.

\begin{tabular}{|c|c|c|}
\hline Praxisauswahlkriterien & Einzelpraxis & Kooperation \\
\hline - Infrastruktur des Orts & - Abstimmungsbedarf $\downarrow$ & $\begin{array}{l}\text { - } \text { Positive } \\
\text { Skaleneffekte * }\end{array}$ \\
\hline Kindergarten & - Reibungsflächen $\downarrow$ & $\begin{array}{l}\text { - Verteilung der } \\
\text { Last }\end{array}$ \\
\hline Schule & - Selbstständigkeit $\uparrow$ & $\begin{array}{l}\text { - finanzielles } \\
\text { Risiko } \downarrow\end{array}$ \\
\hline Einkaufsmöglichkeiten & - Eigener Chef & $\begin{array}{l}\text { - } \text { Kinderbetreu- } \\
\text { ungszeit } \uparrow\end{array}$ \\
\hline $\begin{array}{l}\text { Lebenserhaltungs- } \\
\text { kosten }\end{array}$ & & $\begin{array}{l}\text { - } \text { kein } \\
\text { Einzelkämpfer }\end{array}$ \\
\hline Immobilienpreis & & - Spaßfaktor $\uparrow$ \\
\hline - Heimatnähe & & $\begin{array}{l}\text { - Kollegialer } \\
\text { Austausch } \uparrow\end{array}$ \\
\hline - Wohnortnähe & & $\begin{array}{l}\text { - Vertretungs- } \\
\text { möglichkeit }\end{array}$ \\
\hline \multicolumn{3}{|l|}{ - Stadtnähe } \\
\hline - Nähe zur (Uni)Klinik & & \\
\hline $\begin{array}{l}\text { * Produktionsmenge stei } \\
\text { Produktionsfaktoren stär }\end{array}$ & $\begin{array}{l}\text { im Verhältnis zu den eing } \\
\text { ran [32] }\end{array}$ & tzten \\
\hline
\end{tabular}

gentlich, wusste ich, dass ich da jetzt nicht auf Sand baue, sondern eher auf Stein."(NL4, Z. 110 ff.)

\section{Einzelpraxis oder Kooperation}

Zusammenfassen lässt sich dieser Punkt mit der Frage: Wieweit geht der Wunsch nach Unabhängigkeit und den sich daraus ergebenen Konsequenzen? Während diese in einer Einzelpraxis maximal ausgeprägt sind, stellt eine Kooperation eine Zwischenlösung dar, gewissermaßen einen Hybrid. > Tab. 2 stellt die Vorteile einer Einzelpraxis denen einer Kooperation gegenüber.

„Den kollegialen Austausch, den mag ich. Oder einfach mal abseits des Fachlichen, man muss ja nicht immer über Medizin reden und dann auch einfach, dass sich die Last sich auf mehrere Schultern verteilt. Ganz, ja, typisches Beispiel halt mit Erkrankung oder dann mit Urlaub“. (NL5, Z.181 ff.)

\section{Diskussion}

Die Ergebnisse zeigen, dass die bessere Möglichkeit der Vereinbarkeit von Familie und Beruf sowie die Unzufriedenheit mit dem Klinikalltag gewichtige Punkte für eine Niederlassung sind. Diese zentralen Punkte wurden auch bei einer Studie mit Ärzten in Weiterbildung aus dem Jahr 2017 identifiziert [21].
Weitere Punkte, die wir in der vorliegenden Studie untersucht haben, sind Einflussfaktoren auf den Niederlassungsprozess und auf die Auswahl einer Praxis. Im Prozess der Niederlassung stehen der familiäre Rückhalt, eine professionelle Beratung und der kollegiale Austausch als positive Einflussfaktoren im Vordergrund. Auf der negativen Seite dominieren insbesondere das komplexe Abrechnungssystem, die Regressangst und die hohen anfänglichen Kosten.

Diese Einflussfaktoren auf die Niederlassung lagen auch unter den fünf meistgenannten bei einer Befragung von Ärzten in Weiterbildung aus dem Jahr 2011 [19] und wurden in einer Studie mit Ärzten in Weiterbildung, die im Krankenhaus arbeiteten 2015 erneut bestätigt [22]. Hierbei zeigte sich, dass nur 39\% ihre Arbeitsbedingungen als gut oder sehr gut einstufen. Dabei stellt die Vereinbarkeit mit dem Privatleben das bedeutendste Merkmal für Ärzte in Weiterbildung dar und unterstreicht damit die Bedeutung dieser Faktoren [18, 22].

Der durchschnittliche Zeitaufwand für bürokratische Aufgaben im niedergelassenen Bereich liegt zwischen 1,5 und 2 Stunden pro Tag und Arzt [23]. Im Krankenhaus müssen für bürokratische Aufgaben von $55 \%$ der Ärzte mehr als 2 Stunden am Tag investiert werden [24].

Die Wahl der Praxisform hängt von der Ausprägung des Wunsches nach kompletter Selbstständigkeit und Einzelkämpfernatur im Rahmen einer Einzelpraxis gegenüber einer hybriden Lösung in Form einer Kooperation mit klarem Bekenntnis zu Selbstständigkeit und verstärktem kollegialen Austausch ab. Beim direkten finanziellen Vergleich liegt pro Praxisinhaber der durchschnittliche Jahresüberschuss bei Kooperationen 26\% höher als bei Einzelpraxen, bei gleichzeitig im Durchschnitt 2 Stunden weniger Arbeitsaufkommen pro Woche in einer Kooperation [25].

Die Entscheidung für die Allgemeinmedizin liegt in der Freude an der Arbeit als Generalist, dem Blick für den ganzen Menschen, der hohen Lebensqualität und der ausgeprägten Familienkompatibilität [18].

\section{Strategien}

Ansätze zur Erhöhung der Bereitschaft sich hausärztlich niederzulassen, können an unterschiedlichen Stellen der Aus- und Weiterbildung greifen. Ein zentraler Punkt ist das Hervorheben der Vorteile einer niedergelassenen Tätigkeit als Allgemeinmediziner. Um Studierenden und Ärzten in Weiterbildung den verbesserten Status quo eines niedergelassenen Allgemeinmediziners näher zu bringen, könnte neben einem longitudinalem Kontakt Informationsmaterial mit prägnant aufgelisteten Vorteilen hilfreich sein. Hierbei sollten z. B. die geringe Anzahl an Diensten, die flache Hierarchie, die überdurchschnittlich hohe berufliche Zufriedenheit sowie die Familienkompatibilität hervorgehoben werden. Aus einer Ärzteumfrage aus dem Jahr 2016 geht hervor, dass Hausärzte zu 92 \% zufrieden mit ihrer Arbeit sind. Damit liegen sie überdurchschnittlich hoch im Vergleich zu anderen niedergelassenen Fachärzten. Ähnlich sieht es auch mit der Zufriedenheit der wirtschaftlichen Situation der Praxis aus [26]. Die Arbeitszeit eines niedergelassenen Hausarztes ist seit 2012 sukzessiv gesunken [26] und ist vergleichbar mit der durchschnittlichen Arbeitszeit anderer Vertragsärzte [25]. Zudem sollte man die häufig vorhandenen finanziellen Bedenken ansprechen und sie mit realen Zahlen entschärfen. So lagt 
im Jahr 2017 die Insolvenzrate von Allgemeinmedizinerpraxen unter $0,05 \%$ [27].

Zu den Elementen der „Kompetenzzentren Weiterbildung Allgemeinmedizin“ gehört das „Kompetenzbasierte Curriculum Allgemeinmedizin“. In diesem sind Praxismanagementelemente wie Personalführung und Betriebswirtschaftslehre enthalten [28]. In einigen Bundesländern wird auf dem Boden dieses Curriculums ein die Weiterbildung begleitendes Schulungsprogramm durch die „Kompetenzzentren Weiterbildung Allgemeinmedizin“ angeboten, welches auch Praxismanagementelemente beinhaltet. Dies ist eine wichtige Neuerung im Vergleich zur Situation, dass wirtschaftliche und führungstechnische Elemente kaum oder gar kein Bestandteil des Studiums und der Weiterbildung waren [12, 29].

Bei Studierenden und Ärzten in Weiterbildung wirkt zudem ein kontinuierlicher Kontakt mit dem Fach Allgemeinmedizin durch motivierte Ärzte sowie eine verbesserte praxisnahe Ausbildung [30]. Eine zu schmale Aus- und Weiterbildung hingegen kann dazu führen, sich mit einer geringeren Wahrscheinlichkeit für eine Tätigkeit im ländlichen Raum zu entscheiden [31].

Weitere Elemente um den Niederlassungsprozesses zu optimieren können erfahrene Kollegen als Ansprechpartner und die Betonung der im Vergleich einer Tätigkeit in der Klinik geringeren Belastung durch Bürokratie sein. Des Weiteren bietet eine transparente Darstellung des Niederlassungsprozesses mit den jeweiligen unterstützenden Akteuren zusätzliches Potenzial für zukünftige niedergelassene Allgemeinmediziner. Vorteilhaft wäre hierbei ein standardisiertes Vorgehen mit regionalen und lokalen Adaptionsmöglichkeiten.

Bezüglich der Bürokratie geht es hauptsächlich um deren Reduzierung. Hier wurde unter anderem von der Kassenärztlichen Bundesvereinigung die Forderung nach gesetzlich verpflichteten Abbauzielen für bürokratische Belastung in Arztpraxen gestellt. Ein vergleichbares Abbauziel hatte die Bundesregierung in der Wirtschaft mit einer Reduktion der Bürokratie um $25 \%$ erfolgreich umgesetzt [23].

\section{Stärken und Limitationen der Studie}

Eine der Stärken der Studie besteht in der Auswahl von Ärzten, die sich in den letzten Jahren niedergelassenen haben, da diese den Prozess der Niederlassung zeitnah und aktuell berichten konnten. Durch die vorangegangene Stratifizierung der Teilnehmer konnten Allgemeinärzte aus Stadt wie auch Land erreicht werden.

Die dargestellten Ergebnisse sind methodisch bedingt lediglich als Hypothesen zu verstehen. Aufgrund des Studiendesigns wurde von einer Non-Responder-Analyse abgesehen. Ein positives Selektionsbias aufgrund besonders motivierter Allgemeinmediziner und ausschließlicher Rekrutierung von Ärzten aus dem Förderprogramm Allgemeinmedizin kann nicht ausgeschlossen werden.

\section{Schlussfolgerungen}

Das Bündel an Strategien, um die Niederlassungsbereitschaft zu erhöhen, sollte aus dem Hervorheben der Vorteile der Tätigkeit als niedergelassener Allgemeinmediziner über verschiedene Medien bestehen. Hierunter fallen

- die geringere Anzahl an Diensten,

- die höhere Arbeitszufriedenheit,
- die bessere Familienkompatibilität,

- der höhere Verdienst und

- die geringere Bürokratie

- verglichen mit Ärzten, die im Krankenhaus tätig sind.

Zudem sollte im Rahmen der Aus- und Weiterbildung ein kontinuierlicher \& praxisnaher Kontakt mit der Allgemeinmedizin sichergestellt werden. Lehrangebote zum Praxismanagement sollten ferner ausgebaut werden und der Niederlassungsprozess standardisiert werden. Letztlich könnte eine weitere Reduktion der Bürokratie, die niedergelassene Ärzten bewältigen müssen und eine serviceorientierte Kommunikation zusätzlich zu einer Verbesserung der Niederlassungsbereitschaft beitragen.

Zukünftige Studien sollten beleuchten, ob die Form der Niederlassung in einer Einzelpraxis oder Kooperation sowie rechtliche Rahmenbedingungen Einfluss auf die hemmenden und fördernden Faktoren haben.

\section{Danksagung}

Wir danken Frau Franziska Mennerich für ihre hilfreichen Kommentare zum Manuskript.

Interessenkonflikt

Die Autorinnen/Autoren geben an, dass kein Interessenkonflikt besteht.

Literatur

[1] Gerlach F. Der Hausarzt der Zukunft ist Teamplayer. In: Rexin B, editor. 4/11 ed. https://aok-bv.de: Gesundheit und Gesellschaft; 2011

[2] Augurzky BK, Ingo Fachkräftebedarf im Gesundheits- und Sozialwesen 2030: Gutachten im Auftrag des Sachverständigenrates zur Begutachtung der Gesamtwirtschaftlichen Entwicklung. https://www. sachverstaendigenrat-wirtschaft.de/: Sachverständigenrat zur Begutachtung der Gesamtwirtschaftlichen Entwicklung. 2018; Contract No.: No. 06/2018

[3] OECD. Glossary of statistical terms. https://stats.oecd.org/glossaryOECD Publishing; 2001 [updated 12.11.2001; cited 10.05.2019]

[4] Sundmacher L, Schang L, Schüttig W et al. Gutachten zur Weiterentwicklung der Bedarfsplanung i.S.d. §§ 99 ff. SGB V zur Sicherung der vertragsärztlichen Versorgung 2018

[5] KBV. Statistische Informationen aus dem Bundesarztregister. http:// www.kbv.de: Kassenärztliche Bundesvereinigung, 2014

[6] KBV. Statistische Informationen aus dem Bundesarztregister. http:// www.kbv.de: Kassenärztliche Bundesvereinigung, 2018

[7] Bundesärztekammer. Ärztestatistik zum 31. Dezember 2018. http:// www.bundesaerztekammer.de: Bundesärztekammer, 2019

[8] Stock C, Szecsenyi J, Riedinger-Riebl U et al. Projektion des Bedarfs an hausärztlicher Versorgung auf Gemeindeebene. Gesundheitswesen 2015; 77: 939-946. doi:10.1055/s-0034-1398605

[9] Pötzsch O, Rößger F. Bevölkerung Deutschlands bis 2060. 13. ed. https://www.destatis.de: Statistisches Bundesamt, 2015

[10] Bundesärztekammer. Ärztestatistik zum 31. Dezember 2011. http:// www.bundesaerztekammer.de: Bundesärztekammer, 2012 
[11] Bundesärztekammer. Ärztestatistik zum 31. Dezember 2017. http:// www.bundesaerztekammer.de: Bundesärztekammer, 2018

[12] Jacob R, Kopp J, Schultz S. Berufsmonitoring Medizinstudenten 2014. http://www.kbv.de: Kassenärzliche Bundesvereinigung, 2015

[13] Jäger C, Szecsenyi J, Freund T et al. Developing a tailored intervention: implementing recommendations for polypharmacy in multimorbid patients (PomP). Zeitschrift für Evidenz, Fortbildung und Qualität im Gesundheitswesen 2014; 108: 270-277. doi:10.1016/j.zefq.2013.08.014

[14] Wirtschaftsministerium, Baden-Württemberg Landesentwicklungsplanung 2002 Baden-Württemberg. www.wm.baden-wuerttemberg. de: Wirtschaftsministerium Baden-Württemberg, 2002

[15] Bundesärztekammer. Evaluationsbericht für das Jahr 2011. http:// www.bundesaerztekammer.de: Bundesärztekammer, 2013

[16] Natanzon I, Ose D, Szecsenyi J et al. What factors aid in the recruitment of general practice as a career? An enquiry by interview of general practitioners. Dtsch Med Wochenschr 2010; 135: 10111015. doi:10.1055/s-0030-1253690

[17] Natanzon I, Szecsenyi J, Ose D et al. Future potential country doctor: the perspectives of German GPs. Rural Remote Health 2010; 10: 1347

[18] Roos M, Blauth E, Steinhäuser ] et al. Gebietsweiterbildung Allgemeinmedizin in Deutschland: Eine bundesweite Umfrage unter Ärztinnen und Ärzten in Weiterbildung. Zeitschrift für Evidenz, Fortbildung und Qualität im Gesundheitswesen 2011; 105: 81-88. doi:https://doi. org/10.1016/j.zefq.2010.11.007

[19] Steinhäuser J, Annan N, Roos M et al. Lösungsansätze gegen den Allgemeinarztmangel auf dem Land - Ergebnisse einer Online-Befragung unter Ärzten in Weiterbildung. Dtsch Med Wochenschr 2011; 136: 1715-1719. doi:10.1055/s-0031-1272576

[20] Mayring P. Qualitative Inhaltsanalyse: Grundlagen und Techniken. Beltz; 2015

[21] Barth N, Linde K, Schneider A. Niederlassungsmotive - Die Bereitschaft zur Niederlassung in eigener Praxis von Ärztinnen und Ärzten in Weiterbildung zum Facharzt für Allgemeinmedizin. Gesundheitswesen 2017; 79: 638-644
[22] Flintrop ]. Ärzte in Weiterbildung: Unzufrieden in der Klinik - Respekt vor der Niederlassung. Dtsch Arztebl International 2015; 112: 216

[23] Wittberg V, Kluge H, Rottmann H et al. BIX 2018: Der Bürokratieindex für die vertragsärztliche Versorgung. http://www.kbv.de: 2018

[24] Marburger-Bund. Gesamtauswertung - MB-Monitor 2017. https:// www.marburger-bund.de/: 2017

[25] Zentralinstitut. Jahresbericht 2016: Wirtschaftliche Situation und Rahmenbedingungen in der vertragsärztlichen Versorgung der Jahre 2012 bis 2015. https://www.zi-pp.de: Zentralinstitut für die kassenärztliche Versorgung in Deutschland, 2018

[26] Korzilius H. Ärzteumfrage: Große Zufriedenheit trotz hoher Belastung. Dtsch Arztebl International 2016; 113: 1902-1904

[27] Destastis. Unternehmen und Arbeitsstätten: Insolvenzverfahren. Dezember und Jahr 2017. https://www.destatis.de: 2018

[28] Steinhäuser J. Kompetenzbasiertes Curriculum Allgemeinmedizin. www.degam.de: DEGAM, 2015

[29] Kohlhaas A, Götz K, Berger S et al. Development of a Simulation Game for Teaching Entrepreneurial Skills to Novice Health Professionals in an Interprofessional Learning Environment 2017. 362-369. doi:10.3238/ zfa.2017.0362-0369

[30] Steinhauser J, Miksch A, Hermann K et al. What do medical students think of family medicine? Results of an online cross-sectional study in the federal state of Baden-Wuerttemberg. Dtsch Med Wochenschr 2013; 138: 2137-2142. doi:10.1055/s-0033-1349450

[31] Wilhelmi L, Ingendae F, Steinhaeuser J. What leads to the subjective perception of a 'rural area'? A qualitative study with undergraduate students and postgraduate trainees in Germany to tailor strategies against physician's shortage. Rural Remote Health 2018; 18: 4694. doi:10.22605/rrh4694

[32] Wöhe G, Döring U, Brösel G. Einführung in die Allgemeine Betriebswirtschaftslehre. Vahlen; 2016 